PROCEEDINGS OF THE

AMERICAN MATHEMATICAL SOCIETY

Volume 128, Number 5, Pages 1531-1535

S 0002-9939(99)05364-2

Article electronically published on August 5, 1999

\title{
A K-ENERGY CHARACTERIZATION OF EXTREMAL KÄHLER METRICS
}

\author{
SANTIAGO R. SIMANCA
}

(Communicated by Leslie Saper)

\begin{abstract}
We show that for any polarized compact Kähler manifold, the extremal Kähler metrics that represent the given cohomology class can be characterized as critical points of a suitably defined K-energy functional.
\end{abstract}

Let $(M, J, \Omega)$ be a polarized Kähler manifold of complex dimension $n$. Thus, $(M, J)$ is a complex manifold of Kähler type, and $\Omega$ is a cohomology class in $H^{1,1}$ that can be represented by the Kähler form of a Kähler metric. One may hope to find a canonical metric that represents $\Omega$ by studying critical points of a suitable Riemannian functional. Since the set $\Omega^{+}$of Kähler metrics of fixed Kähler class $\Omega$ is parametriced by (an open set of) functions, Calabi [2, 3] proposed the functional

$$
\begin{aligned}
\Omega^{+} & \longrightarrow \mathbb{R} \\
\omega & \mapsto \int_{M} \mathbf{s}_{\omega}^{2} d \mu_{\omega} .
\end{aligned}
$$

Here $\mathbf{s}_{\omega}$ and $d \mu_{\omega}$ are the scalar curvature and volume form of $\omega$, respectively. Calabi computed the Euler-Lagrange equation, and showed that the critical metrics of this functional are those for which the gradient of the scalar curvature is a realholomorphic vector field. He called these metrics extremal.

Inspired by the work of Donaldson on Yang-Mills connections on stable bundles, Mabuchi [7] introduced the K-energy functional on $\Omega^{+}$for polarized manifolds with positive first Chern class. The critical points of this functional are Kähler Einstein metrics, and it has played a significant role in the study of these metrics for this type of manifolds [1, 4]. The purpose of this note is to show that extremal Kähler metrics can also be defined as critical points of a functional analogous to the K-energy.

Let $G$ be a maximal compact subgroup of the biholomorphism group of $(M, J)$, and let $g$ be a Kähler metric on $M$ with Kähler class $\Omega$. Without loss of generality, we assume that $g$ is $G$-invariant. We denote by $L_{k, G}^{2}$ the real Hilbert space of $G$ invariant real-valued functions of class $L_{k}^{2}$, and consider $G$-invariant deformations of this metric preserving the Kähler class:

$$
\tilde{\omega}=\omega+i \partial \bar{\partial} \varphi, \quad \varphi \in L_{k+4, G}^{2}, k>n .
$$

In this expression, the condition $k>n$ ensures that $L_{k, G}^{2}$ is a Banach algebra, making the scalar curvature of $\tilde{\omega}$ a well-defined function in the space.

Received by the editors June 22, 1998.

1991 Mathematics Subject Classification. Primary 58E11; Secondary 53C55, 53C25.

Key words and phrases. Extremal Kähler metrics, K-energy.

The author was partially supported by the Gabriella and Paul Rosenbaum Foundation. 
Recall that $\mathbb{C} \otimes T M$ decomposes into the $\pm i$-eigenspaces of $J$,

$$
\mathbb{C} \otimes T M=T^{1,0} M \oplus T^{0,1} M .
$$

This decomposition induces a splitting of sections of this bundle as vector fields of type $(1,0)$ and $(0,1)$, respectively. In fact, we may express any tensor field in terms of the corresponding decomposition $\bigotimes^{r} \mathbb{C} \otimes T M=\bigoplus_{p+q=r} \otimes^{p} T^{1,0} M \otimes \bigotimes^{q} T^{0,1} M$.

Given any Kähler metric $\tilde{g}$, a smooth complex-valued function $f$ gives rise to a vector field of type $(1,0)$ by the rule $f \mapsto \partial^{\#} f=\partial_{\tilde{g}}^{\#} f$, where $\partial_{\tilde{g}}^{\#} f:=(\bar{\partial} f)^{\#}$ is the type $(1,0)$ piece of the gradient of $f$ with respect to $\tilde{g}$. This vector field is not, in general, holomorphic. For that, we need to require that $f$ satisfies the fourth-order equation

$$
\left(\bar{\partial} \partial^{\#}\right)^{*} \bar{\partial} \partial^{\#} f=\frac{1}{4} \Delta^{2} f+\frac{1}{2} \tilde{\mathbf{r}}^{\mu \nu} \nabla_{\mu} \nabla_{\nu} f+\frac{1}{2}\left(\nabla^{\bar{\ell}} \tilde{\mathbf{s}}\right) \nabla_{\bar{\ell}} f=0,
$$

where the adjoint, Ricci tensor $\tilde{\mathbf{r}}$, scalar curvature $\tilde{\mathbf{s}}$ and other relevant quantities are those for the metric $\tilde{g}$.

Every complex-valued solution $f$ of (2) is therefore associated with a holomorphic vector field $\Xi=\partial^{\#} f$, and since the operator is elliptic, the space of such functions is finite dimensional. However, since $\left(\bar{\partial} \partial^{\#}\right)^{*} \bar{\partial} \partial^{\#}$ is not a real operator, in general, the real and imaginary part of a solution will not be solutions. It has been proven elsewhere [6] that if $f$ is a real-valued solution of this equation, then the imaginary part of $\partial^{\#} f$ is a Killing field of $\tilde{g}$, and that a Killing field arises in this way if, and only if, it has a zero somewhere on the manifold.

Let $\mathfrak{z} \subset \mathfrak{g}$ denote the center of $\mathfrak{g}$, the Lie algebra of $G$, and let $\mathfrak{z}_{0}=\mathfrak{z} \cap \mathfrak{g}_{0}$, where $\mathfrak{g}_{0} \subset \mathfrak{g}$ is the ideal of Killing fields which have zeroes. If $\tilde{g}$ is any $G$-invariant Kähler metric on $(M, J)$, then each element of $\mathfrak{z}_{0}$ is of the form $J \operatorname{grad} f$ for a real-valued solution of (21). In fact, $\mathfrak{z}_{0}$ corresponds to the set of real solutions $f$ which are invariant under $G$, since

$$
\partial^{\#}: \operatorname{ker}\left[\left(\bar{\partial} \partial_{\tilde{g}}^{\#}\right)^{*} \bar{\partial} \partial_{\tilde{g}}\right] \rightarrow \mathfrak{h}_{0}
$$

is a homomorphism of $G$-modules. Here, $\mathfrak{h}_{0}$ is the ideal of vector fields with zeroes in the Lie algebra $\mathfrak{h}(M)$ of the group of biholomorphism of $(M, J)$.

The restriction of $\operatorname{ker}\left(\bar{\partial} \partial_{\tilde{g}}^{\#}\right)^{*} \bar{\partial} \partial_{\tilde{g}}$ to $L_{k+4, G}^{2}$ depends smoothly on the $G$-invariant metric $\tilde{g}$. Indeed, choose a basis $\left\{X_{1}, \ldots, X_{m}\right\}$ for $\mathfrak{z}_{0}$, and, for each $(1,1)$-form $\chi$ on $(M, J)$, consider the set of functions

$$
\begin{aligned}
& p_{0}(\chi)=1, \\
& p_{j}(\chi)=2 i \mathbf{G} \bar{\partial}_{g}^{*}\left(\left(J X_{\jmath}+i X_{\jmath}\right) \downarrow \chi\right), \quad j=1, \ldots, m,
\end{aligned}
$$

where $\mathbf{G}$ is the Green's operator of the metric $g$. If $\tilde{\omega}$ is the Kähler form of the $G$-invariant metric $\tilde{g}$, then $\partial_{\tilde{g}}^{\#} p_{j}(\tilde{\omega})=J X_{\jmath}+i X_{\jmath}$, and the set $\left\{p_{j}(\tilde{\omega})\right\}_{\jmath=0}^{m}$ consists of real-valued functions and form a basis for $\operatorname{ker}\left(\bar{\partial} \partial_{\tilde{g}}^{\#}\right)^{*} \bar{\partial} \partial_{\tilde{g}}$. Furthermore, for metrics $\tilde{\omega}$ as in (1), the map $\varphi \mapsto p_{j}(\omega+i \partial \bar{\partial} \varphi)$ is, for each $j$, bounded as a linear map from $L_{k+4, G}^{2}$ to $L_{k+3, G}^{2}$.

With respect to the fixed $L^{2}$ inner product, let $\left\{f_{\tilde{\omega}}^{0}, \ldots, f_{\tilde{\omega}}^{m}\right\}$ be the orthonormal set extracted from $\left\{p_{j}(\tilde{\omega})\right\}$ by the Gram-Schmidt procedure. We then let

$$
\begin{aligned}
\pi_{\tilde{\omega}}: L_{k, G}^{2} & \rightarrow L_{k, G}^{2} \\
u & \mapsto \sum_{j=0}^{m}\left\langle f_{\tilde{\omega}}^{j}, u\right\rangle_{L^{2}} f_{\tilde{\omega}}^{j}
\end{aligned}
$$


denote the associated projector. In fact, by the regularity of the functions $\left\{p_{1}, \ldots\right.$, $\left.p_{m}\right\}$, this projection can be defined on $L_{k+j, G}^{2}$ for $j=0,1,2,3$, and for metrics as in (11), the map $\varphi \mapsto \pi_{\tilde{\omega}}$ is smooth from a suitable neighborhood of the origin in $L_{k+4, G}^{2}$ to the real Hilbert space $\operatorname{End}\left(L_{k+j, G}^{2}\right) \cong \bigotimes^{2} L_{k+j, G}^{2}$.

Let us denote by $\wedge_{k, G}^{1,1}$ the space of real forms of type $(1,1)$, invariant under $G$ and of class $L_{k}^{2}$.

Proposition 1. Given any $G$-invariant metric $\tilde{g}$, there exists a unique continuous linear map

$$
\Pi_{\tilde{g}}: \wedge_{k+2, G}^{1,1} \mapsto \wedge_{k+2, G}^{1,1},
$$

which intertwines the trace and the projection map $\pi_{\tilde{\omega}}$ in (3), and such that $\eta-\Pi_{\tilde{g}} \eta$ is cohomologous to zero for all $\eta \in \wedge_{k+2, G}^{1,1}$. For metrics $\tilde{\omega}$ as in (1), the map $\varphi \mapsto \Pi_{\tilde{\omega}}$ from $L_{k+4, G}^{2}$ to $\operatorname{End}\left(\wedge_{k+2, G}^{1,1}\right)$ is smooth.

Proof. Let $\eta \in \wedge_{k+2, G}^{1,1}$. Since $\Pi_{\tilde{\omega}} \eta$ must be of the form $\eta+i \partial \bar{\partial} f$ for some real-valued function $f$, the intertwining property of the projection and trace gives that

$$
\operatorname{trace}_{\tilde{\omega}} \eta-\frac{1}{2} \Delta_{\tilde{\omega}} f=\pi_{\tilde{\omega}} \operatorname{trace}_{\tilde{\omega}} \eta
$$

and so

$$
\Delta_{\tilde{\omega}} f=-2\left(\pi_{\tilde{\omega}}-1\right) \operatorname{trace}_{\tilde{\omega}} \eta .
$$

The right side of this expression is a $G$-invariant real-valued function in the complement of the constants. We can then solve the equation for $f$ and obtain a real-valued solution which is invariant under $G$. By the continuity properties of the map $\pi_{\tilde{\omega}}$, for metrics as in (1) the map $\varphi \mapsto \Pi_{\tilde{\omega}}$ is a smooth map from a suitable neighborhood of the origin in $L_{k+4, G}^{2}$ to the real Hilbert space $\operatorname{End}\left(\wedge_{k+2, G}^{1,1}\right)$.

We now adapt ideas of T. Mabuchi 7] to produce another characterization of extremal Kähler metrics. Mabuchi was interested in the study of Kähler Einstein metrics on manifolds with positive first Chern class. These metrics have constant positive scalar curvature, and therefore, are extremal in the sense of Calabi. However, more generally, an extremal metric on the polarized Kähler manifold $(M, J, \Omega)$ is a Kähler metric that represents the class $\Omega$, and for which the gradient of the scalar curvature is a real-holomorphic vector field, that is, $\partial_{\omega}^{\#} \mathbf{s}_{\omega}$ is a holomorphic vector field 1 Metrics of constant scalar curvature are extremal, but after Calabi's initial result 2, there are now many known examples of extremal metrics which do not have constant scalar curvature 6].

For the polarized Kähler manifold $(M, J, \Omega)$ and maximal compact subgroup $G$, we define

$$
\mathfrak{M}_{\Omega, G}=\{\omega: \omega \text { is Kähler, } G \text {-invariant and }[\omega]=\Omega\} .
$$

Given any two metrics $\omega_{0}$ and $\omega_{1}$ in $\mathfrak{M}_{\Omega, G}$, there exists a $G$-invariant function $\varphi$, unique modulo constants, such that

$$
\omega_{1}=\omega_{0}+i \partial \bar{\partial} \varphi
$$

\footnotetext{
${ }^{1}$ Though our notation doesn't indicate it, the vector field $\partial_{\omega}^{\#} \pi_{\omega} \mathbf{s}_{\omega}$ only depends on $\Omega$ and not on the particular metric $\omega$ in $\Omega^{+}$chosen to represent it 5]. If $\omega$ is extremal, this vector field coincides with $\partial_{\omega}^{\#} \mathbf{s}_{\omega}$.
} 
Let $\varphi(t)$ be a curve of $G$-invariant functions such that $\omega(t)=\omega_{0}+i \partial \bar{\partial} \varphi(t) \in \mathfrak{M}_{\Omega, G}$ and $\omega(0)=\omega_{0}, \omega(1)=\omega_{1}$. We set

$$
M\left(\omega_{0}, \omega_{1}\right)=-\int_{0}^{1} d t \int_{M} \dot{\varphi}(t)(\mathbf{s}(t)-\pi(t) \mathbf{s}(t)) d \mu(t),
$$

where $\mathbf{s}(t)$ and $d \mu(t)$ are the scalar curvature and volume form of the metric $\omega(t)$, $\pi(t)$ is the projection (3) onto the space of $G$-invariant holomorphic potentials associated with this metric, and $\dot{\varphi}(t)=\frac{d \varphi}{d t}(t)$. We now see how the argument in 7] adapts to obtain the following

Lemma 1. The definition of $M$ above is independent of the curve $t \rightarrow \varphi(t)$. Furthermore, $M$ satisfies the cocycle conditions $M\left(\omega_{0}, \omega_{1}\right)=-M\left(\omega_{1}, \omega_{0}\right)$ and $M\left(\omega_{0}, \omega_{1}\right)+M\left(\omega_{1}, \omega_{2}\right)+M\left(\omega_{2}, \omega_{0}\right)=0$.

Proof. Let $\rho(t)$ be the Ricci form of the metric $\omega(t)$. By Proposition 1 the $2 n$ form $(\mathbf{s}(t)-\pi(t) \mathbf{s}(t)) d \mu(t)$ is equal to $2 n(\rho(t)-\Pi(t) \rho(t)) \wedge \omega^{n-1}(t)$, and therefore closed. This closed form can be thought of as a closed 1-form on $\mathfrak{M}_{\Omega, G}$. The definition of $M\left(\omega_{0}, \omega_{1}\right)$ is nothing but the line integral of this one-form from $\omega_{0}$ to $\omega_{1}$. The rest of the proof is straightforward.

Fix any Kähler metric $\omega_{0}$ in $\mathfrak{M}_{\Omega, G}$ and define the K-energy function by

$$
\begin{gathered}
\mu_{\omega_{0}}: \mathfrak{M}_{\Omega, G} \longrightarrow \mathbb{R} \\
\mu_{\omega_{0}}(\omega)=M\left(\omega_{0}, \omega\right) .
\end{gathered}
$$

Proposition 2. Let $\omega_{t}=\omega_{0}+i \partial \bar{\partial} \varphi_{t}$ be a one-parameter family of smooth Kähler metrics in $\mathfrak{M}_{\Omega, G}$. Then,

$$
\frac{d}{d t} \mu_{\omega_{0}}\left(\omega_{t}\right)=\int_{M} \nabla_{t} \dot{\varphi}_{t}\left(f_{t}\right) d \mu_{t}
$$

where $f_{t}$ is the function, unique up to a constant, determined by the relation

$$
\rho_{t}=\Pi_{t} \rho_{t}+i \partial \bar{\partial} f_{t} .
$$

Here $\rho_{t}$ and $\Pi_{t}$ are, respectively, the Ricci form and projection (4) associated to $\omega_{t}$.

Proof. By the previous lemma, it follows that

$$
\frac{d}{d t} \mu_{\omega_{0}}\left(\omega_{t}\right)=-\int_{M} \dot{\varphi}_{t}\left(\mathbf{s}_{t}-\pi_{t} \mathbf{s}_{t}\right) d \mu_{t}
$$

Using the properties of the projection $\Pi_{t}$, we see that the integrand above can be written as $\dot{\varphi}_{t} \Delta_{t} f_{f}$. The result is now a consequence of integration by parts.

Theorem 1. Let $(M, J, \Omega)$ be a compact polarized Kähler manifold, and $G$ a maximal compact subgroup of the biholomorphism group of $(M, J)$. A metric is extremal iff it is a critical point of the K-energy $\mu_{\omega_{0}}$ functional, calculated by fixing an arbitrary reference metric $\omega_{0} \in \mathfrak{M}_{\Omega, G}$. In that case, the identity component of its isometry group is $G$.

Of course, by the results of Calabi [3] we know that two extremal metrics, whose isometries groups have identity components equal to $G$ and $G^{\prime}$, are conjugated by a biholomorphism which conjugates the subgroups $G$ and $G^{\prime}$. 
Proof. By the previous proposition, if $\omega(t)=\omega_{0}+i \partial \bar{\partial} \varphi(t)$ is an arbitrary curve in $\mathfrak{M}_{\Omega, G}$ such that $\omega(0)=\omega \in \mathfrak{M}_{\Omega, G}$, then

$$
\left.\frac{d}{d t} \mu_{\omega_{0}}(\omega(t))\right|_{t=0}=\int_{M} \nabla_{\omega} \dot{\varphi}(0)\left(f_{\omega}\right) d \mu_{\omega}
$$

where $f_{\omega}$ is a function such that

$$
\rho_{\omega}=\Pi_{\omega} \rho_{\omega}+i \partial \bar{\partial} f_{\omega} .
$$

Thus, $\omega$ is a critical point of $\mu_{\omega_{0}}$ if and only if the function $f_{\omega}$ is a constant. But then

$$
\rho_{\omega}=\Pi_{\omega} \rho_{\omega},
$$

which implies that $\omega$ is extremal [2] because, when computing the contraction with $\omega$, we obtain

$$
\mathbf{s}_{\omega}=\pi_{\omega} \mathbf{s}_{\omega}
$$

and so the vector field $\partial_{\omega}^{\#} \mathbf{s}_{\omega}$ is holomorphic.

The natural question to ask is if the functional $\mu_{\omega_{0}}$ is proper (in the sense of Tian (4), and furthermore, if this leads to an a-priori $C^{0}$ estimate for the Kähler potential of an extremal Kähler metric $\tilde{\omega}=\omega_{0}+i \partial \bar{\partial} \varphi$ in the class $\Omega$. Even if one assumes the existence of an extremal metric representing this class, it is not clear how to generalize the argument of Bando and Mabuchi 11 to prove that $\mu_{\omega_{0}}$ is bounded below. The Monge-Ampère equation that one must solve to deform $\omega_{0}$ to an extremal metric is given by

$$
\frac{\operatorname{det}\left(g_{\imath \bar{\jmath}}+\varphi_{\imath \bar{\jmath}}\right)}{\operatorname{det}\left(g_{\imath \bar{\jmath}}\right)}=e^{-c \varphi+f+\tilde{\mathbf{G}}\left(\pi_{\tilde{g}} \mathbf{s}_{\tilde{g}}-2 \operatorname{trace}_{\tilde{g}} \rho^{\perp}\right)} .
$$

Here $c$ is a constant whose sign is equal to the sign of the total scalar curvature, the metric $\tilde{g}$ is given by the Kähler form $\tilde{\omega}=\omega_{0}+i \partial \bar{\partial} \varphi, \tilde{\mathbf{G}}$ is its Green's operator, and the Ricci curvature of $\omega_{0}$ is written as $\rho_{0}=c \omega_{0}+\rho^{\perp}+i \partial \bar{\partial} f$ with $\rho^{\perp}$ a $\omega_{0}$ trace-free harmonic $(1,1)$ form. It remains to be seen what geometric conditions could lead to the successful analysis of this equation.

\section{REFERENCES}

[1] S. Bando \& T. Mabuchi, Uniqueness of Einstein Kähler metrics modulo connected group actions, In Adv. Studies in Pure Math. 10, Algebraic Geometry Sendai 1985 (ed. T. Oda) (1986), pp. 11-40. MR 89c:53029

[2] E. Calabi, Extremal Kähler Metrics, in Seminar on Differential Geometry (ed. S.T. Yau) Princeton, 1982. MR 83i:53088

[3] E. Calabi, Extremal Kähler Metrics II, in Differential Geometry and Complex Analysis (ed. I. Chavel and H.M. Farkas), Springer-Verlag, 1985. MR 86h:53067

[4] W. Ding \& G. Tian, Kähler-Einstein metrics and the generalized Futaki invariant, Invent. Math. 110 (1992) pp. 315-335. MR 93m:53039

[5] A. Futaki, Kähler-Einstein Metrics and Integral Invariants, Lect. Notes in Math., 1314, Springer-Verlag. MR 90a:53053

[6] C. LeBrun and S.R. Simanca, Extremal Kähler Metrics and Complex Deformation Theory, Geom. and Funct. Anal. 4 (1994), pp. 298-336. MR 95k:58041

[7] T. Mabuchi, K-energy maps integrating Futaki invariants, Tohoku Math. Journal. 38 (1986), pp 575-593. MR 88b:53060

Department of Applied Mathematics, State University of New York at Stony Brook, Stony Brook, New York 11794-3600

E-mail address: santiago@ams.sunysb.edu 